\title{
ANALYSIS OF IMPACT CHATTERING
}

\author{
M. AKHMET AND S. ÇAĞ
}

Received 29 September, 2016

\begin{abstract}
In this paper, mechanical models with Newton's Law of impacts are studied. One of the most interesting properties in some of these models is chattering. This phenomenon is understood as the appearance of an infinite number of impacts occurring in a finite time. Conclusion on the presence of chattering is made exclusively by examination of the right hand side of impact models for the first time. Criteria for the sets of initial data which always lead to chattering are established. The Moon-Holmes model is subject to regular impact perturbations for the chattering generation. Using the chattering solutions, continuous chattering is generated. To depress the chattering, Pyragas control is applied. Illustrative examples are provided to demonstrate the impact chattering.
\end{abstract}

2010 Mathematics Subject Classification: 34A37; 34D10

Keywords: impact mechanism, the Moon-Holmes model, chattering, bouncing bead, Pyragas control

\section{INTRODUCTION}

The implementation of sliding mode control is often irritated by high frequency oscillations known as "chattering" in system outputs issued by dynamics from actuators and sensors ignored in system modeling [11]. In study [10], chattering is considered as a special type of oscillation characterized by very small amplitudes that are decreasing with time. In impacting systems, it is understood as an infinite number of discontinuity moments occurring in a finite time period, for instance, a ball bouncing to rest on a horizontal surface [7]. It is asserted in [7] that chattering resembles with the inelastic collapse. The balls dissipate their energy through an infinite number of collisions in a finite time interval. Budd and Dux [5] showed that chattering can occur for a periodically forced, single degree of freedom impact oscillator with a restitution law. They demonstrated that chattering can form part of a periodic motion, and this relates to certain types of chaotic behavior. However, they studied through an example. Using the solution, they proved the existence of chattering for a linear system.

Nordmark and Piiroinen [14] considered simulation problems for chattering as well as analysis of stability of the limit cycle, which is chattering by solving the first 
variational equations. Moreover, they used the mappings, which are constructed with the help of a solution, in simulation schemes. Similar to the one in paper [5], it was shown that the existence of chattering for a linear system. Nonetheless, in both papers $[5,14]$, they do not consider the conditions which guarantee the appearance of chattering. In this study, we consider the chattering as a motion with infinite number of discontinuities in a finite time. This is the first time that sufficient conditions are provided for the chattering based on properties not on maps derived with the help of solutions, but, on conditions for the right-hand side of impulsive systems. Our models essentially are nonlinear (see, for example, Example 1). Since this is the first result in this direction, the models under consideration are respectively simple. Nevertheless, this is a class of mechanical models which can be significantly enlarged in the future investigations by consideration of large ensembles of impact oscillators and weakening conditions of the present paper. We consider models with vibrating surface of impacts as well as analyzed problems of Pyragas controllability and existence of continuous chattering for a model connected unilaterally to a system with an impact chattering. An interesting problem of the regular perturbation of a system with chattering is discussed.

A particular feature of system with impacts is the existence of the chattering. We have two different types of it, namely complete and incomplete chattering [5, 14]. Complete chattering is the phenomenon wherein a system an infinite number of discontinuities in a finite time occurs, where the velocity tends to zero uniformly. Incomplete chattering bears on a sequence of the impacts that initially has the same behavior as complete chattering, but it ends after a large but finite number of impacts [14]. In section 3, we will discuss the transient chattering for systems with small parameter considering the transformation of the incomplete chattering to the complete one when the parameter diminishes to zero.

It was first found by Arnold [4] that the significant characteristic property of chatter vibration is that it is not generated by external periodic forces, but rather it is generated in the dynamic process itself. Therefore, it is important to emphasize that the systems under investigation in this paper are autonomous.

Consider the problem of impact interaction of a body falling in the uniform gravity force field with a fixed horizontal base. After colliding with the base the body bounces back with the velocity whose norm is equal to the norm of the pre-impact velocity multiplied by $r$, where $r$ is the restitution coefficient, $0<r<1$. Then, after some time interval the body will fall on the base again and the norm of its velocity will be equal to the norm of bouncing velocity in the previous collision multiplied by $r$. The process cannot end in a finite number of collisions. Thus, the considered phenomenon consists in following: after the initial collision a series of repeated collisions of attenuated to zero, which ends in a finite time with establishing a long contact between interacted bodies. Arising this contact results in decreasing number 
of degrees of freedom of the system by a unit or more. So, it is reasonable to call this phenomenon the impact chattering.

It is shown by investigations and observations that the impact chattering meets in operating almost every mechanism and machine of impact-oscillating type [13]. Various problems of impact chattering are far from trivial, and their solutions cannot be obtained in closed form for rather general case. As for the use of approximate analytical and numerous methods, it is simplified essentially if one proceeds from the conception about infinite number of impacts inside a finite time range. For example, the existence of impact chattering was investigated in [13]. They simply consider the free falling of a bead on an immobile base and on a vibrating table with constant velocity. In this paper, we consider a more general system and prove the existence of impact chattering.

The chattering phenomena are unwanted in engineering since it is an appearance of infinite discontinuities in a short period of time and this makes theoretical analysis of mechanical models difficult. We have a research plan to consider theoretical and mathematical complexities connected to chattering, and we approach the problem from one of the two possible points of view. The first one is when mechanical models change such that the theoretical chattering disappears [1]. The other point of view, which is considered in this paper, is that we approximate a model with infinite moments of discontinuities with those having a finite number of impacts.

This article is organized as follows. First of all, the impact model is stated. In this model each collision is assumed instantaneous, and it comes to rest after an infinite number of impulse moments in a finite time. The existence of chattering is proved. Asymptotic approximation of solutions with chattering are discussed in section 3 . Then, we show that the chattering occurs for a bead bouncing on a sinusoidally vibrating table in section 4 . The modified Moon-Holmes model with a small perturbation is discussed in section 5. Using the continuous dependence on parameters and initial value for the impulsive differential equations with non-fixed moments, it is shown that the solution of the modified Moon-Holmes model is chattering. Following that, the appearance of continuous chattering by perturbation method is demonstrated in section 6. Finally, by Pyragas control method the chattering solution is controlled to be periodic.

\section{EXISTENCE OF CHATTERING}

An impacting system admits a chattering if there is a solution with infinite impulse moments in a finite time. Moreover, we will say that a perturbed system admits a transient chattering, if a number of impacts increases to infinity on a fixed interval as the small parameter tends to zero. 
A mechanism with a rigid flat surface of impacts and the constant coefficient of restitution $r, 0<r<1$, can be modeled by the following impulsive system

$$
\begin{aligned}
\ddot{x} & =f(x, \dot{x}), \\
\left.\Delta \dot{x}\right|_{x=\varphi} & =-(1+r) \dot{x},
\end{aligned}
$$

where $x(t)$ is the coordinate of the bead which is over the impact surface $x=\varphi$, $\dot{x}(t)$ is its velocity, $f(u, v)$ is a continuous function on the domain $H=\{0<\varphi \leq$ $u \leq h,|v| \leq \bar{h}\}$ for fixed positive numbers $h, \bar{h}$, and it satisfies the local Lipschitz condition in its variables on $H$. The equality $\Delta \dot{x}(\theta)=\dot{x}(\theta+)-\dot{x}(\theta-)$ denotes the jump operator in which $t=\theta$ is the time when the bead reaches the rigid obstacle, $\dot{x}(\theta-)$ is the pre-impact velocity and $\dot{x}(\theta+)$ is the post-impact velocity.

In system (2.1), we need the following conditions.

(C1) There is a positive number $m$ such that $f(u, v)<-m$ for all $(u, v) \in H$,

(C2) $f(u, v)=f(u,-v)$ for all $(u, v) \in H$.

Conditions on function $f(u, v)$ and compactness of domain $H$ imply that there exists a positive number $M$ such that $f(u, v) \geq-M$ for all $(u, v) \in H$.

Theorem 1. If conditions (C1), (C2) are satisfied and the following inequality

$$
M \sqrt{\frac{2(h-\varphi)}{m}}<\bar{h}
$$

is valid, then all solutions with initial value $(x(0), \dot{x}(0))=\left(x_{0}, 0\right), \varphi<x_{0}<h$, of system (2.1) are chattering.

Proof. Consider an initial value $\left(x_{0}, 0\right) \in H, \varphi<x_{0}<h$. Denoting $x_{1}=x, x_{2}=\dot{x}$ present the system (2.1) as

$$
\begin{aligned}
\dot{x_{1}} & =x_{2}, \\
\dot{x_{2}} & =f\left(x_{1}, x_{2}\right), \\
\left.\Delta x_{2}\right|_{x_{1}=\varphi} & =-(1+r) x_{2} .
\end{aligned}
$$

The solution of system (2.3) starting at $\left(x_{0}, 0\right)$ is

$$
\begin{gathered}
x_{1}(t)=x_{0}+\int_{0}^{t}(t-s) f\left(x_{1}(s), x_{2}(s)\right) d s, \\
x_{2}(t)=\int_{0}^{t} f\left(x_{1}(s), x_{2}(s)\right) d s,
\end{gathered}
$$

while it is continuous. By equation (2.4a) and condition $(C 1)$, the coordinate $x_{1}(t)$ decreases to $\varphi$ such that there exists a moment $\theta_{1}$ where $x_{1}\left(\theta_{1}\right)=\varphi$ and $x_{2}\left(\theta_{1}\right)<0$. Moreover, $x_{1}\left(\theta_{1}+\right)=\varphi$ and $x_{2}\left(\theta_{1}+\right)=-r x_{2}\left(\theta_{1}\right)>0$.

Let us show that the solution is continuable to $+\infty$ and it remains in the domain $H$. First of all, consider the interval $\left[0, \theta_{1}\right]$. From conditions $(\mathrm{C} 1)$ and $(\mathrm{C} 2)$, it implies 
that $x_{1}(t) \leq x_{0}<h, t \in\left[0, \theta_{1}\right]$. Using (2.4a) and inequality $\varphi<x_{0}<h$ we get $|h-\varphi|>\left|\varphi-x_{0}\right|=\left|\int_{0}^{\theta_{1}}\left(\theta_{1}-s\right) f\left(x_{1}(s), x_{2}(s)\right) d s\right| \geq \int_{0}^{\theta_{1}}\left(\theta_{1}-s\right) m d s=m \frac{\theta_{1}^{2}}{2}$,

which implies that $\theta_{1}<\sqrt{\frac{2(h-\varphi)}{m}}$.

Consequently, from (2.4b) and condition (2.2)

$$
\left|x_{2}\left(\theta_{1}\right)\right|=\left|\int_{0}^{\theta_{1}} f\left(x_{1}(s), x_{2}(s)\right) d s\right| \leq M \theta_{1}<\bar{h} .
$$

Thus, we obtain that $\varphi \leq x_{1}(t)<h$ and $\left|x_{2}(t)\right|<\bar{h}$ for $t \in\left[0, \theta_{1}\right]$.

Applying the same arguments as for $\theta_{1}$ one can show that there is an intersection moment $\theta_{2}$ such that $x_{1}\left(\theta_{2}\right)=\varphi$ and $x_{1}(t)>\varphi, t \in\left(\theta_{1}, \theta_{2}\right)$. In this interval, we have

$$
\begin{gathered}
x_{1}(t)=\varphi+x_{2}\left(\theta_{1}+\right)\left(t-\theta_{1}\right)+\int_{\theta_{1}}^{t}(t-s) f\left(x_{1}(s), x_{2}(s)\right) d s, \\
x_{2}(t)=x_{2}\left(\theta_{1}+\right)+\int_{\theta_{1}}^{t} f\left(x_{1}(s), x_{2}(s)\right) d s,
\end{gathered}
$$

By condition (C2), $x_{2}\left(\theta_{1}+\right)$ is the maximum value of $\left|x_{2}(t)\right|$ for $t \in\left(\theta_{1}, \theta_{2}\right]$. Thus, $\left|x_{2}(t)\right| \leq r\left|x_{2}\left(\theta_{1}\right)\right|<r \bar{h}<\bar{h}$. Moreover, from conditions $(C 1)$ and $(C 2)$, there exists a moment $\xi_{1}, \theta_{1}<\xi_{1}<\theta_{2}$, such that $x_{2}\left(\xi_{1}\right)=0$ and $x_{1}\left(\xi_{1}\right)$ is the maximum value of $x_{1}(t)$ on $\left(\theta_{1}, \theta_{2}\right]$. Thus, $x_{1}(t) \leq x_{1}\left(\xi_{1}\right)<x_{0}<h$, and the trajectory of $x(t)$ is in $H$ for $t \in\left[\theta_{1}, \theta_{2}\right]$. Next, recursively, it can be shown that there exists an increasing sequence $\theta_{i}, i=1,2, \ldots$, such that $x_{1}\left(\theta_{i}\right)=\varphi, i=1,2, \ldots$, and the orbit of $x(t)$ is in $H$ for all $t \geq 0$.

Now, we will show that the sequence $\theta_{i}$ converges. The solution of system (2.3) is defined by

$$
\begin{gathered}
x_{1}(t)=\varphi+x_{2}\left(\theta_{i}+\right)\left(t-\theta_{i}\right)+\int_{\theta_{i}}^{t}(t-s) f\left(x_{1}(s), x_{2}(s)\right) d s, \\
x_{2}(t)=x_{2}\left(\theta_{i}+\right)+\int_{\theta_{i}}^{t} f\left(x_{1}(s), x_{2}(s)\right) d s .
\end{gathered}
$$

on the interval $\left(\theta_{i}, \theta_{i+1}\right], i=1,2, \ldots$

Using condition (C1), it can be shown that there exists a moment $\xi_{i}, \theta_{i}<\xi_{i}<$ $\theta_{i+1}$, such that $x_{2}\left(\xi_{i}\right)=0$. Also, utilizing condition (C2), we obtain $\xi_{i}=\frac{\theta_{i}+\theta_{i+1}}{2}$. 
The solution on the interval $\left(\theta_{i+1}, \theta_{i+2}\right]$ is

$$
\begin{aligned}
& x_{1}(t)=\varphi+r x_{2}\left(\theta_{i}+\right)\left(t-\theta_{i+1}\right)+\int_{\theta_{i+1}}^{t}(t-s) f\left(x_{1}(s), x_{2}(s)\right) d s, \\
& x_{2}(t)=r x_{2}\left(\theta_{i}+\right)+\int_{\theta_{i+1}}^{t} f\left(x_{1}(s), x_{2}(s)\right) d s .
\end{aligned}
$$

From $x_{2}\left(\xi_{i}\right)=0$ and $x_{2}\left(\xi_{i+1}\right)=0$, we get

$$
\begin{array}{r}
x_{2}\left(\theta_{i}+\right)=-\int_{\theta_{i}}^{\xi_{i}} f\left(x_{1}(s), x_{2}(s)\right) d s, \\
r x_{2}\left(\theta_{i}+\right)=-\int_{\theta_{i+1}}^{\xi_{i+1}} f\left(x_{1}(s), x_{2}(s)\right) d s .
\end{array}
$$

Let us divide (2.9) by (2.8) in order to get

$$
r=\frac{\int_{\theta_{i+1}}^{\xi_{i+1}} f\left(x_{1}(s), x_{2}(s)\right) d s}{\int_{\theta_{i}}^{\xi_{i}} f\left(x_{1}(s), x_{2}(s)\right) d s} .
$$

Using mean value theorem, we have

$$
r=\frac{\left(\xi_{i+1}-\theta_{i+1}\right) f\left(x_{1}\left(s^{*}\right), x_{2}\left(s^{*}\right)\right)}{\left(\xi_{i}-\theta_{i}\right) f\left(x_{1}\left(s^{* *}\right), x_{2}\left(s^{* *}\right)\right)},
$$

for some $s^{* *}$ and $s^{*}$ in $\left(\theta_{i}, \theta_{i+1}\right)$ and $\left(\theta_{i+1}, \theta_{i+2}\right)$ respectively.

Then,

$$
\frac{\theta_{i+2}-\theta_{i+1}}{\theta_{i+1}-\theta_{i}}=\frac{\xi_{i+1}-\theta_{i+1}}{\xi_{i}-\theta_{i}}<\frac{M_{i}}{m_{i}} r, i=1,2,3 \ldots .
$$

where $M_{i}=\max _{\left[\theta_{i}, \theta_{i+1}\right]}\left|f\left(x_{1}(t), x_{2}(t)\right)\right|$ and $m_{i}=\min _{\left[\theta_{i}, \theta_{i+1}\right]}\left|f\left(x_{1}(t), x_{2}(t)\right)\right|$. Since $r<$ 1, $\max _{\left[\theta_{i}, \theta_{i+1}\right]}\left|x_{1}(t)\right| \rightarrow \varphi$ and $\max _{\left[\theta_{i}, \theta_{i+1}\right]}\left|x_{2}(t)\right| \rightarrow 0$ as $i \rightarrow \infty$. Moreover, continuity of $f(u, v)$ implies that $\frac{M_{i}}{m_{i}} \rightarrow 1$ as $i \rightarrow \infty$. This and (2.11) prove the convergence. The theorem is proved.

Example 1. Consider the following non-linear system

$$
\begin{gathered}
\ddot{x}+\cos (\dot{x})+x^{3}=0, \\
\left.\Delta \dot{x}\right|_{x=2}=-(1+r) \dot{x},
\end{gathered}
$$

in the domain $2 \leq x \leq 2.5,|\dot{x}|<7$. We have $f(x, \dot{x})=-\cos (\dot{x})-x^{3} \leq-7, f(x, \dot{x})=$ $f(x,-\dot{x}), \quad f(x, \dot{x}) \geq-16.625$ in the domain. Condition (2.2) is true since $16.625 \sqrt{1 / 7} \approx 6.28<7$. That is, we are in circumstances of Theorem 1 and if we choose $r=0.8, x(0)=2.1, \dot{x}(0)=0$, the solution of system (2.12) is chattering. The simulation of this solution can be seen in Figure 1 . 

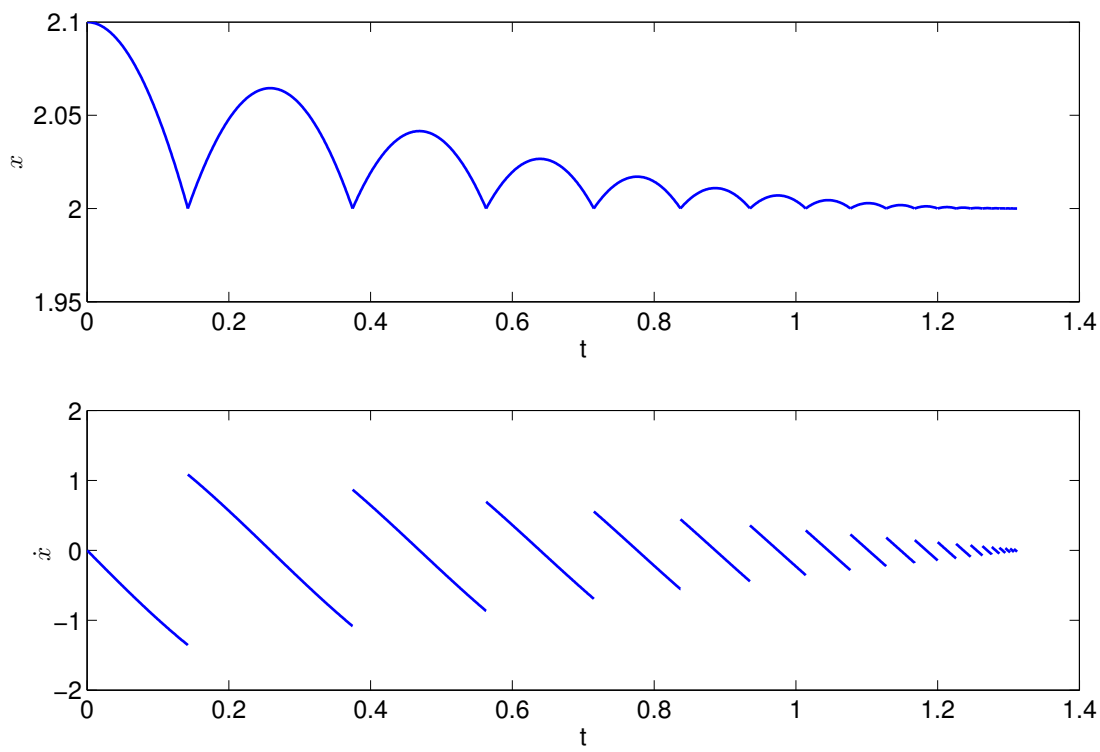

FIGURE 1. The graphs of coordinates $x(t)$ and $\dot{x}(t)$ with initials $x(0)=2.1$ and $\dot{x}(0)=0$ of system (2.12) with $r=0.8$.

\section{ASYMPTOTICS}

Solutions of the system (2.1) admit infinitely many jumps, and this makes, in general, impossible to find an exact solution or adequately to simulate it. So, in this section we suggest considering degenerate equation to find the perturbed system approximately. In order to increase the precision of approximation we follow the idea of asymptotic approximations. Consider the system

$$
\begin{gathered}
\ddot{x}=f(x, \dot{x}), \\
\Delta \dot{x} \mid \begin{array}{c}
x=\varphi \\
i<\left[\frac{1}{r}\right]
\end{array}=-(1+r) \dot{x},
\end{gathered}
$$

where $i$ is the index of impacts $\theta_{i}$, [.] denotes the greatest integer function, with additional condition that the number of impulsive moments has to be not more than $\left[\frac{1}{r}\right]$, i.e., $\theta_{i}, i=1,2, \ldots,\left[\frac{1}{r}\right]$. One can guarantee for the fixed value of the parameter $r$, the incomplete chattering occurs only. The number of impacts increases unboundedly as the parameter tends to zero. For this reason, we say that system (3.1) admits the transient chattering. Assume that this system satisfy all conditions of Theorem 1 . For time $t>\theta_{\left[\frac{1}{r}\right]}$, the system is only governed by $\ddot{x}=f(x, \dot{x})$. Condition $(C 1)$ implies that on the interval $\left[\theta_{\left[\frac{1}{r}\right]}, \theta_{\infty}\right]$ the bead stays on the position $x=\varphi$. 
For each its solution, system (3.1) has finite number of discontinuity moments. That is why, one can find an exact solution of the problem or at least it is possible to make proper simulations. One can easily see that solutions of the last system and system (2.1) with identical initial data coincide on the interval $\left[0, \theta_{\left[\frac{1}{r}\right]}\right)$. They are different only in the interval $\left[\theta_{\left[\frac{1}{r}\right]}, \theta_{\infty}\right]$. The length of the last interval diminishes to 0 as $r \rightarrow 0$. Consequently, the solutions of system (3.1) are asymptotic approximations for the solutions of system (2.1).

\section{THE DYNAMICS OF REPEATED IMPACTS AGAINST A SINUSOIDALLY VIBRATING TABLE}

In this section, we consider a mechanical model consisting of a bead bouncing on a vibrating table, which is investigated in the papers of Holmes and Guckenheimer [8,9]. It is demonstrated that the model can generate chaos [9]. In this paper, we show that in the mechanism one can observe another type of complex dynamics, namely chattering.

Consider a bouncing bead colliding with a sinusoidally vibrating table. Assume that the table is so massive that it does not react to collisions with the bouncing bead and it moves according to law $X(t)=X_{0} \sin \omega t$. The change of the velocity of the bouncing bead at the impact moment is given by the relation $r=\frac{\dot{X}_{+}-\dot{x}_{+}}{\dot{x}_{-}-\dot{X}_{-}}$, where $r$ is the restitution coefficient, $0<r<1, \dot{X}_{-}, \dot{X}_{+}, \dot{x}_{-}, \dot{x}_{+}$are the velocities of the table and the bouncing bead before and after impact, respectively. Since the collision does not affect the velocity of the table, we can write $\dot{X}_{-}=\dot{X}_{+}$. Then the model will be as follows

$$
\begin{aligned}
\ddot{x} & =-g, \\
\left.\Delta \dot{x}\right|_{x=X} & =-(1+r)(\dot{x}-\dot{X}), \\
X(t) & =X_{0} \sin (\omega t),
\end{aligned}
$$

where $g$ is the gravitational acceleration $\left(g \approx 9.8 \mathrm{~m} / \mathrm{s}^{2}\right)$.

Now, let us consider a general form. Instead of gravitational constant $g$, take a function $f(u, v)$. Then, the model will be of the form

$$
\begin{aligned}
\ddot{x} & =f(x, \dot{x}), \\
\left.\Delta \dot{x}\right|_{x=X} & =-(1+r)(\dot{x}-\dot{X}), \\
X(t) & =X_{0} \sin \omega t,
\end{aligned}
$$

where function $f(u, v)$ is a continuous function on the domain $G=\left\{X_{0} / 10 \leq u \leq\right.$ $h,|v| \leq \bar{h}\}$, for fixed positive numbers $h, \bar{h}$, and it satisfies the local Lipschitz condition in its variables on $G$. Also, this system has conditions (C1), (C2) defined in the first section for all $(u, v) \in G$. By conditions on function $f(u, v)$ and compactness of the domain $G$, we have a positive number $M$ such that $f(u, v) \geq-M$ for all $(u, v) \in G$. 
Next, consider the graph of the function $X(t)=X_{0} \sin \omega t$. The slope of the graph is $\dot{X}(t)=X_{0} \omega \cos \omega t$. It is easily seen that if $\omega$ is small and $t$ is near $\pi / 2 \omega$ the graph is close to a horizontal line. Consequently, for sufficiently small $\omega$ and for time $t$ near $\pi / 2 \omega$ if the following inequality

$$
M \sqrt{\frac{2\left(h-X_{0} / 10\right)}{m}}<\bar{h},
$$

is true and conditions $(\mathrm{C} 1),(\mathrm{C} 2)$ are satisfied, according to Theorem 1 there is chattering for solutions whose integral curves are near to the point $P\left(\pi / 2 \omega, X_{0}\right)$. Finally, to demonstrate the result through simulation, we continue with the bouncing bead on the sinusoidally vibrating table.

Example 2. Let us return to the bouncing bead on the sinusoidally vibrating table with the same properties of system (4.2). Then the model will be as follows

$$
\begin{aligned}
& \ddot{x}=-g, \\
& \left.\Delta \dot{x}\right|_{x=X}=-(1+r)(\dot{x}-\dot{X}), \\
& X(t)=X_{0} \sin (\omega t),
\end{aligned}
$$

where $t \geq 0$. Let us take $\varphi=X_{0}=1$ and consider the domain $0.1 \leq x \leq 2,|\dot{x}|<7$. Then, we have $f(x, \dot{x})=-g<0,|f(x, \dot{x})|=|-g|=g=M=m$ and $M \sqrt{\frac{2\left(h-X_{0} / 10\right)}{m}}=\sqrt{37.24}<7$. If we choose the initial conditions $x(2 \pi / \omega)=1.9$, $\dot{x}(2 \pi / \omega)=0$, where $r=0.9, \omega=0.29$, it can be seen that the conditions of Theorem 1 are satisfied and consequently, this solution is chattering. In Figure 2, one can observe the coordinates of system (4.4) which supports our theoretical result.
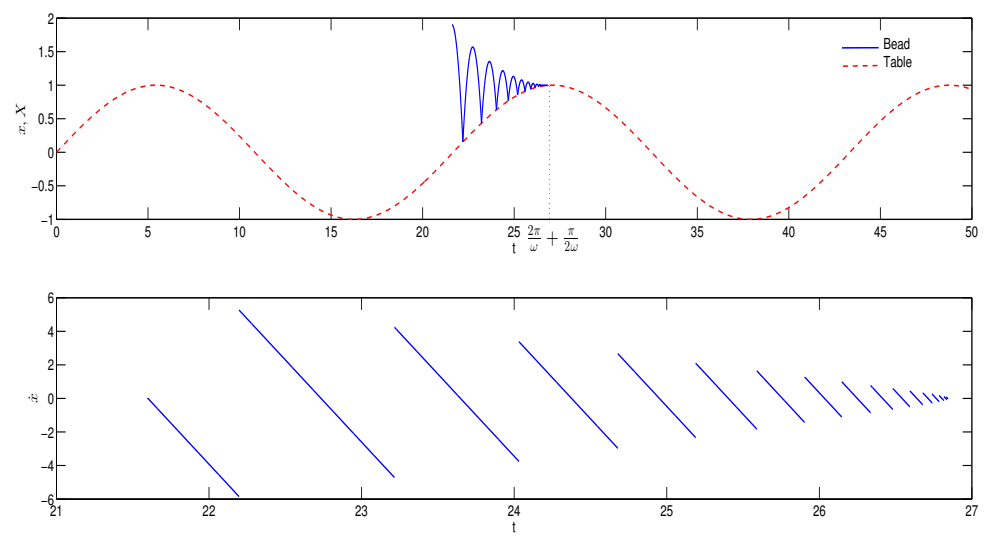

FIGURE 2. The graph of the coordinates of system (4.4). 


\section{The MOdified MoON-Holmes Model}

The main task of this section is to consider the modified Moon-Holmes Model. Moon and Holmes [12] showed that the Duffing equation in the form

$$
\ddot{x}+\delta \dot{x}-x+x^{3}=\gamma \cos w t
$$

provides the simplest possible model for the forced vibrations of a cantilever beam in the nonuniform field of two permanent magnets. Such an equation describes the dynamics of a buckled beam or plate when only one mode of vibration is considered. We modify the model as adding a rigid obstacle over the magnet and in front of the beam such that the beam collides the obstacle and from Newton Law of impacts it bounces back. (The system is sketched in Figure 3.) The suggested model has the form of the following impulsive system

$$
\begin{gathered}
\ddot{x}=-\delta \dot{x}+x-x^{3}+\gamma \cos w t, \\
\left.\Delta \dot{x}\right|_{x=\varphi}=-(1+r) \dot{x},
\end{gathered}
$$

where $x$ is the distance from the wall to the end of the beam, $\varphi$ is the position of the obstacle, $r$ is the restitution coefficient. Now, if the coefficients $\gamma$ and $\delta$ are equal to zero, one obtains

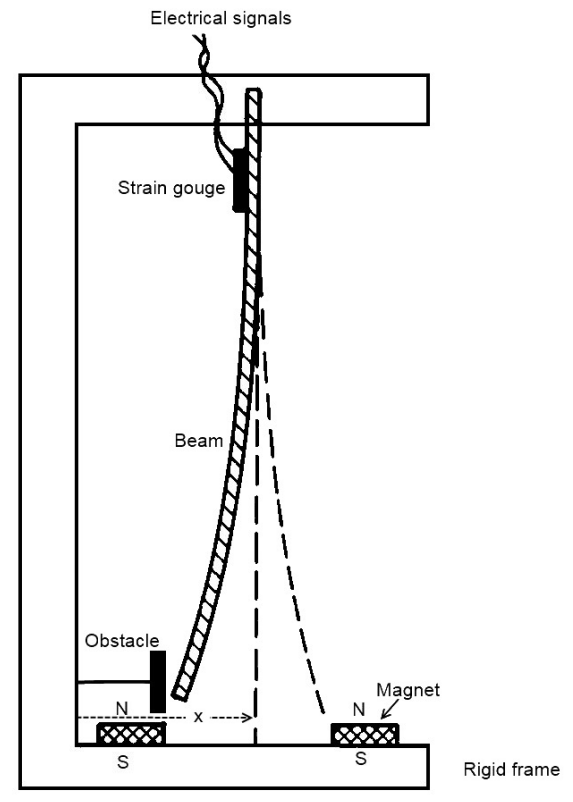

FIGURE 3. The magneto-elastic beam with the obstacle. 


$$
\begin{gathered}
\ddot{x}=x-x^{3}, \\
\left.\Delta \dot{x}\right|_{x=\varphi}=-(1+r) \dot{x} .
\end{gathered}
$$

For this system, choose $\varphi=1.1$ and for the domain $H$ let $h=1.5, \bar{h}=3$. One can see that function $f(x, \dot{x})=x-x^{3}$ satisfies conditions (C1) and (C2), i.e. $-1.875 \leq$ $f(u, v) \leq-0.331$ for all $(u, v) \in H$ and $f(u, v)$ is an even function in $v$. Moreover, condition (2.2) is valid since $1.875 \sqrt{\frac{2(1.5-1.1)}{0.331}} \approx 2,91<3$. Therefore, by Theorem 1 all solutions of system (5.2) with initial values $(x(0), \dot{x}(0))=\left(x_{0}, 0\right), \varphi<x_{0}<h$, are chattering. Obviously, system (5.1) does not satisfy condition (C2). But, one can easily notice that for sufficiently small $\delta$ and $\gamma$, by the continuous dependence on parameters and initial value for the impulsive differential equations with nonfixed moments [2], the solutions of (5.1) with the same initial conditions of (5.2) are chattering as well. For the numerical simulation, let $(x(0), \dot{x}(0))=(1.3,0)$ and $r=0.9$. Then, one can see that Figure 4 supports our theoretical discussion.
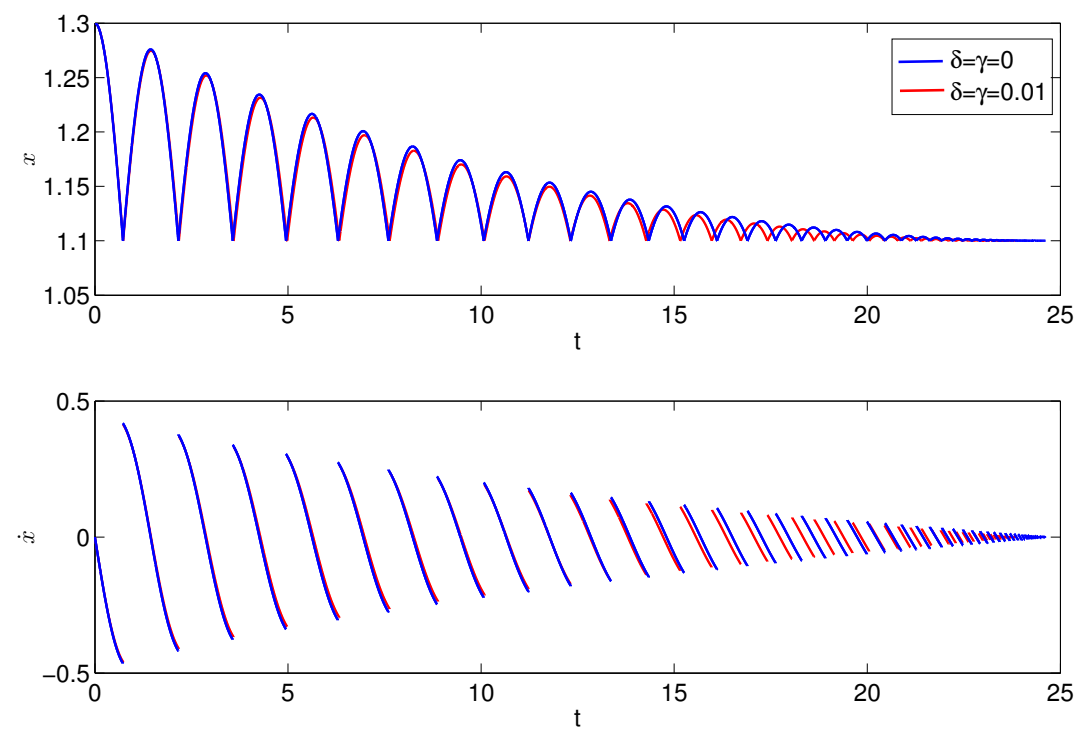

FIgURE 4. The coordinates of systems (5.1) and (5.2) with $w=0.1$. It can be seen that the solution of perturbed system (5.1) is also chattering. 


\section{CONTINuOus CHATtering}

In this section, we demonstrate the continuous chattering which is understood as infinitely many oscillations in finite time. Let us observe how continuous chattering appears if a mechanical model is perturbed with a discontinuous one. For this reason, we couple system (2.1) with the following equation of a mass-spring-damper equation

$$
m \ddot{y}+c \dot{y}+k y=0,
$$

with mass $m$, spring constant $k$, and viscous damper of damping coefficient $c$. If the characteristic equation of system (6.1) has roots with negative real parts, then it admits asymptotically stable equilibrium. By the argument of periodicity theorem for system with stable equilibrium, one can expect that in system (6.1), continuous chattering appears if it is perturbed by a chattering solution of (2.1). Thus, let us write the coupled system taking $f(x, \dot{x})=-g$ in (2.1), $m=1, c=3, k=2$ in (6.1) and $x=x_{1}, \dot{x}=x_{2}, y=x_{3}, \dot{y}=x_{4}$, in the form

$$
\begin{aligned}
& \dot{x}_{1}=x_{2}, \\
& \dot{x}_{2}=-g, \\
& \left.\Delta\right|_{x_{1}=1}=-(1+r) x_{2} \\
& \dot{x}_{3}=x_{4}, \\
& \dot{x}_{4}=-2 x_{3}-3 x_{4}+20 x_{2}^{2} .
\end{aligned}
$$

with initial conditions $x_{1}(0)=6, x_{2}(0)=0, x_{3}(0)=10, x_{4}(0)=-1000$ and $r=0.9$.
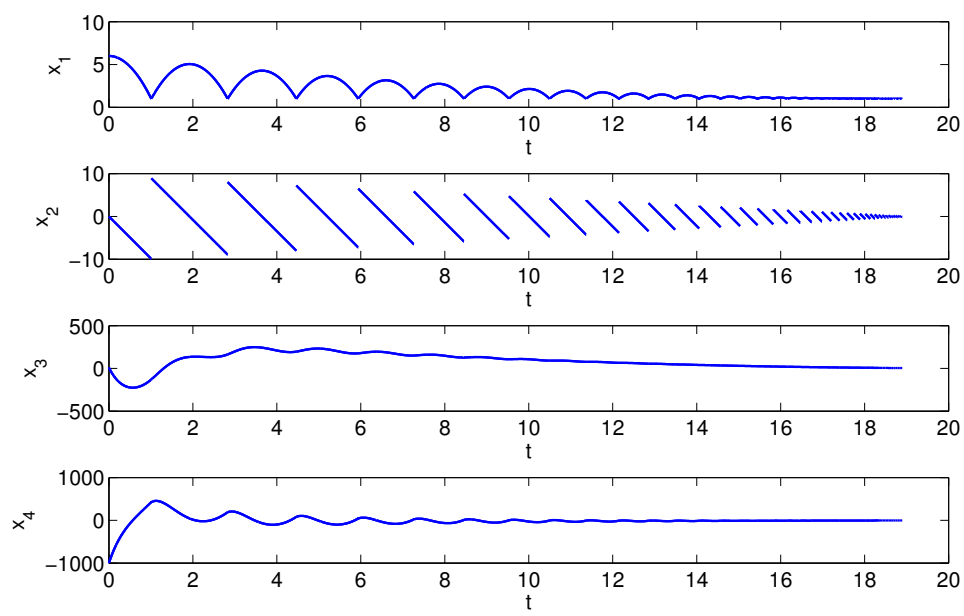

FIGURE 5. The graphs of the coordinates of system (6.2). 
Since the first coupling is unilateral, the second equation does not influence the first one. That is why, its dynamics are the same as in Figure 5. But, for the second coupling in Figure 5, we can see the effect of perturbation which we call as continuous chattering.

\section{PYRAGAS CONTROL}

There are many papers which are searching methods to minimize and control different types of chattering [3,6]. The problem definitely has to be analyzed for the impact chattering, also. One can accept that the control of impact chattering is a concrete perturbation, which brings the system under control to a regular motion. That is, equilibria or periodic motions. In the circumstances of the present research, it is desired that a family of chattering solutions has to be regularized, if not all of them. We will discuss, in this part of the paper, system (2.12) of Example 1. It was shown that any solution of this system, which starts in a domain, is chattering. Let us apply the control of the form $C\left[x_{1}(t-\tau)-x_{1}(t)\right]$ to the system. It is applied, for instance, to stabilize periodic motions of chaotic dynamics, and it is called Pyragas control [15]. Now, we will apply the control to depress the chattering in the system. Let us construct the following system denoting $x_{1}=x$ and $x_{2}=\dot{x}$

$$
\begin{aligned}
& \dot{x_{1}}=x_{2}, \\
& \dot{x_{2}}=-x_{1}^{3}-\cos x_{2}+C\left[x_{1}(t-\tau)-x_{1}(t)\right] \\
& \left.\Delta x_{2}\right|_{x_{1}=2}=-(1+r) x_{2} .
\end{aligned}
$$

We performed a series of simulations of the system with fixed $C=-30, \tau=1$ and $r=0.6$. Consider $x_{2}(0)$ as it was requested to prove family of chattering solutions in Theorem 1. For the initial first coordinate $x_{1}(0)$ we tried values starting from 2.5 to 200.

\begin{tabular}{l|c|c|c|c|c|c}
$x_{1}(0)$ & 2.5 & 3 & 5 & 10 & 100 & 200 \\
\hline Period $T$ & 1.22 & 1.22 & 1.22 & 1.22 & 1.22 & 1.22 \\
\hline Amplitude & 2.933 & 2.933 & 2.933 & 2.933 & 2.933 & 2.933
\end{tabular}

TABLE 1. Periods and amplitudes of the first coordinate $x_{1}(t)$ of system (7.1) for different values of $x_{1}(0)$.

For all these solutions the ultimate periodicity has been approved with period $T=$ 1.22. Observe that the period is different from the delay term $\tau=1$. One can see from the table that the amplitudes are equal to 2.933 for all values of $x_{1}(0)$ as well. At the same time, the chattering has not been decaying for the solution with $x_{1}(0)=2.1$. These all demonstrate that the control problem can be solved for the chattering, but certain conditions have to be determined to specify the controllable domains and 
conditions for the stability of the arranged periodic motions. We suppose that these problems will be researched in next papers.

For $x_{1}(0)=3$, the periodic orbit $x_{1}(t)$ can be seen in Figure 6, which shows the effectiveness of the control.

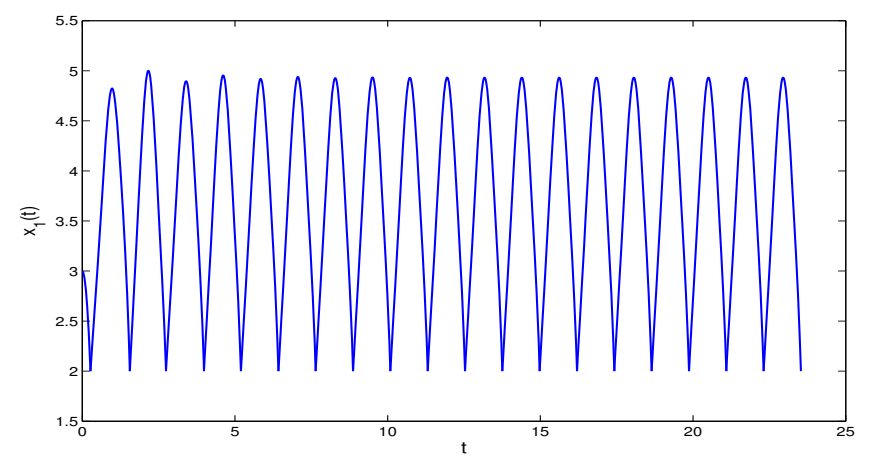

FIGURE 6. Simulation of the first coordinate $x_{1}(t)$ of controlled system (7.1) with initial conditions $x_{1}(0)=3, x_{2}(0)=0$.

\section{CONCLUSion}

In this article, we have considered the mechanical models with impacts. For these models, the chattering phenomenon, which is defined as a motion with infinitely many discontinuities in a finite time, is studied. The sufficient conditions are determined for the existence of the chattering. Asymptotics are discussed to find an approximation solution and to simulate the chattering solution. We study the famous example: the bouncing bead on a sinusoidally vibrating table which generates chaos [9]. It is shown that this mechanism has chattering solutions. Furthermore, we modify the Moon-Holmes model [12], which yields chaos also, with an obstacle to obtain an impacting model. We demonstrate that this model provides chattering. Perturbing a continuous mechanical process by a discontinuous one having chattering solutions, continuous chattering, which is defined as the appearance of infinitely many oscillations in a finite time, is constructed.

The application of results of paper [14] is to prove the existence of a unique chattering solution of the bouncing ball, see Section 3.5. At the same time, by simulation it is proven that a double pendulum admits chattering. Our method, in some sense, is more wider than the one in paper [14]. For example, by Theorem 1 in this paper, we have verified that there are infinitely many chattering motions with initial values in an interval. Thus, the present result is complement to that one accomplished in [14]. However, our approach does not work for the double pendulum, since condition $(C 2)$ is not valid for the model. Nevertheless, in our next investigation, we plan to extend the method without condition $(C 2)$. 


\section{REFERENCES}

[1] M. U. Akhmet and A. Kıvılcım, "The Models with Impact Deformations," Discontinuity, Nonlinearity, and Complexity, vol. 4, no. 1, pp. 49-78, 2015.

[2] M. Akhmet, Principles of Discontinuous Dynamical Systems. New York: Springer, 2010.

[3] A. Alasty and H. Salarieh, "Controlling the chaos using fuzzy estimation of $\{\mathrm{OGY}\}$ and Pyragas controllers," Chaos, Solitons \& Fractals, vol. 26, no. 2, pp. 379-392, 2005, doi: 10.1016/j.chaos.2004.12.034.

[4] R. N. Arnold, "Cutting Tools Research: Report of Subcommittee on Carbide Tools: The Mechanism of Tool Vibration in the Cutting of Steel," Proceedings of the Institution of Mechanical Engineers, vol. 154, no. 1, pp. 261-284, 1946.

[5] C. Budd and F. Dux, "Chattering and Related Behaviour in Impact Oscillators," Philosophical Transactions: Physical Sciences and Engineering, vol. 347, no. 1683, pp. pp. 365-389, 1994, doi: 10.1098/rsta.1994.0049.

[6] W.-C. Chen, "Dynamics and control of a financial system with time-delayed feedbacks," Chaos, Solitons \& Fractals, vol. 37, no. 4, pp. 1198-1207, 2008, doi: 10.1016/j.chaos.2006.10.016.

[7] S. Giusepponi, F. Marchesoni, and M. Borromeo, "Randomness in the bouncing ball dynamics," Physica A: Statistical Mechanics and its Applications, vol. 351, no. 1, pp. 142-158, 2005, doi: 10.1016/j.physa.2004.12.016.

[8] J. Guckenheimer and P. Holmes, Nonlinear oscillations, dynamical systems, and bifurcations of vector fields, ser. Applied mathematical sciences. Springer-Verlag, 1990.

[9] P. J. Holmes, "The dynamics of repeated impacts with a sinusoidally vibrating table," Journal of Sound and Vibration, vol. 84, no. 2, pp. 173-189, 1982, doi: 10.1016/S0022-460X(82)80002-3.

[10] R. A. Ibrahim, Vibro-Impact Dynamics: Modeling, Mapping and Applications, ser. Lecture Notes in Applied and Computational Mechanics. Springer, 2009.

[11] H. Lee and V. I. Utkin, "Chattering suppression methods in sliding mode control systems," Annual Reviews in Control, vol. 31, no. 2, pp. 179-188, 2007, doi: 10.1016/j.arcontrol.2007.08.001.

[12] F. C. Moon and P. J. Holmes, "A magnetoelastic strange attractor," J. Sound Vib., vol. 65, no. 2, pp. 275-296, 1979, doi: 10.1016/0022-460X(79)90520-0.

[13] R. F. Nagaev and E. B. Kremer, Mechanical Processes with Repeated Attenuated Impacts. World Scientific, 1999.

[14] A. B. Nordmark and P. T. Piiroinen, "Simulation and stability analysis of impacting systems with complete chattering," Nonlinear Dynamics, vol. 58, no. 1-2, pp. 85-106, 2009, doi: 10.1007/s11071-008-9463-y.

[15] K. Pyragas, "Continuous control of chaos by self-controlling feedback," Physics Letters A, vol. 170, no. 6, pp. 421-428, 1992, doi: 10.1016/0375-9601(92)90745-8.

\section{Authors' addresses}

\section{Akhmet}

Middle East Technical University, Department of Mathematics, Ankara, Turkey

E-mail address: marat @metu.edu.tr

S. Çă̆

Middle East Technical University, Department of Mathematics, Ankara, Turkey

E-mail address: scag@metu.edu.tr 